\title{
Les nouveaux partenariats famille-école au Québec : l'extériorité comme stratégie de survie des familles défavorisées?
}

\author{
New Family-School Partnerships in Quebec : Exteriority as a \\ Survival Strategy for Disadvantaged Families? \\ Las nuevas cooperaciones entre la familia y la escuela en el \\ Quebec : ¿ la exterioridad como la estrategia de supervivencia \\ de las familias desfavorecidas?
}

\section{Michèle Vatz-Laaroussi}

Numéro 35, printemps 1996

Familles et école

URI : https://id.erudit.org/iderudit/005245ar

DOI : https://doi.org/10.7202/005245ar

Aller au sommaire du numéro

\section{Éditeur(s)}

Lien social et Politiques

\section{ISSN}

1204-3206 (imprimé)

1703-9665 (numérique)

\section{Découvrir la revue}

\section{Citer cet article}

Vatz-Laaroussi, M. (1996). Les nouveaux partenariats famille-école au Québec : l'extériorité comme stratégie de survie des familles défavorisées ? Lien social et Politiques, (35), 87-97. https://doi.org/10.7202/005245ar
Résumé de l'article

Dans la société québécoise de la fin du vingtième siècle, le partenariat entre les familles et l'école est posé à la fois comme une question de société et comme un préalable a toute évolution de l'institution scolaire. Par l'analyse de données de plusieurs recherches menées d'abord en France puis au Québec auprès de familles dites de milieu défavorisé, nous proposons ici de distinguer deux types de stratégies mises en œuvre par les écoles québécoises dans leur fonction éducative, pour ensuite comprendre les pratiques des familles de milieu défavorisé grâce à l'élaboration d'un inventaire des stratégies familiales de survie. L'arrimage entre stratégies d'école et stratégies familiales de survie sera notre analyseur de la position des familles par rapport à l'école. Cette position, selon nous, repose sur une logique d'extériorité qui est le seul gage de la survie matérielle et symbolique tant de l'entité familiale que de chacun des membres de cette entité. 


\section{Les nouveaux partenariats famille-école au Québec : l'extériorité comme stratégie de survie des familles défavorisées?}

\section{Michèle Vatz Laaroussi}

Le présent texte s'articule sur l'hypothèse que les rapports entre les familles et l'école se construisent à travers des stratégies mises en cuvre, d'une part par l'institution scolaire dans ses processus de scolarisation et de partenariat, d'autre part par les familles dans les usages qu'elles font du système éducatif. Afin d'argumenter cette perspective et suivant en cela l'approche développée par Lessard (1994), nous proposons une démarche d'analyse du partenariat familles-école. Après une brève présentation des enjeux de ces par-

LIEN SOCIAL ET POLITIQUES - RIAC, 35 PRINTEMPS 1996 tenariats, nous identifierons, grâce à l'analyse de programmes implantés dans des écoles primaires du Québec, deux stratégies partenariales de l'école ainsi que leurs effets attendus et pervers, spécifiquement vis-à-vis des classes sociales les plus défavorisées. Nous appuyant sur nos recherches auprès de familles de ces milieux, d'abord en France, puis au Québec, nous élaborerons ensuite le concept de stratégies familiales de survie et l'illustrerons par trois stratégies de survie mises en œuvre par les familles québécoises de milieux défavorisés pour, à la fois, s'adapter à leur situation et résister à ce que nous envisageons comme une forme d'impérialisme de la logique scolaire dans la vie des enfants et de leurs parents. Nous argumenterons finalement que les stratégies de ces familles reposent sur une rationalité d'extériorité vis-à-vis de l'école, gage de survie symbolique de l'entité familiale et de chacun de ses membres.

\section{La famille et l'école : un partenariat sous contrainte}

Au Québec, institutions familiale et scolaire ont longtemps partagé la responsabilité de l'éducation des enfants sous la toutepuissance de l'Église catholique. Les deux instances procédaient à l'intérieur d'un même tout gouverné par les valeurs, normes et 

défavorisées?

règles de la religion (Carisse, 1974). Les années 1960 , par le processus de la Révolution tranquille, ont, d'un même élan, entraîné famille et école sur la voie des grands changements. La famille a vu diminuer le code religieux qui la régulait ainsi que se modifier les référents sociaux, moraux et éducatifs sur lesquels elle reposait, pour aller finalement vers une désinstitutionnalisation (Roussel, 1989). Par le «virage familial», les années 1990 lui redonnent une place importante sur les plans socio-économique et politique: celle de la famille responsable, fournissant services, support et protection à ses divers membres, mais aussi celle de la famille partenaire (Bouchard, 1991; Politique de la santé et du bien-être du Québec, 1992), née à la fois de la fin de l'État providence, à tout le moins sur le plan économique, et de l'idéologie de l'expertise qui découpe le champ social par compétences, expertises, besoins spécifiques et facteurs de risques (Dandurand, 1995). Dans ce morcellement du social par spécificités, la famille est ainsi construite et définie par de multiples partenariats mais aussi contrainte à collaborer avec tous les experts qui l'entourent. En particulier, les experts de l'enfance et de l'éducation qui se retrouvent dans l'institution scolaire deviennent partenaires obligés des familles. Dans le même temps, pour les diverses institutions sociales, dont l'école, la famille remplit une fonction essentielle puisque, par sa seule existence partenariale, elle permet souvent de légitimer et de renforcer les stratégies d'intervention sanitaires, sociales et éducatives.

L'école, pour sa part, bien que demeurant confessionnelle, s'est vu attribuer nombre de valeurs, de discours, d'idéologies et de modalités pédagogiques issus de l'univers de la laiicité. Un des résultats essentiels des profondes mutations qu'elle a vécues est sans conteste l'augmentation de son accessibilité et du niveau moyen de scolarisation des Québécois(e)s (Lessard, 1994). Tout au long de ces transformations, deux enjeux sont restés constants pour l'institution scolaire. Il s'agit d'abord et toujours de la moindre performance et du décrochage précoce accru chez les élèves des milieux les plus défavorisés (Proulx, 1994). Il est ensuite question, tant dans les discours provenant de l'école (Conseil supérieur de l'éducation, 1994) que dans ceux des organismes familiaux (Conseil de la famille, 1995), du rapport entre l'école et les familles comme point d'ancrage de la réussite des élèves. Dès 1991, la ministre déléguée à la famille du Québec s'interrogeait sur la place de la famille dans l'éducation scolaire des enfants et sur les moyens à mettre en œuvre pour stimuler un partenariat école-familles. En 1995, les États généraux sur l'éducation amènent les Québécois à interroger les résultats obtenus par la politique scolaire et éducative des trente dernières années ainsi qu'à reconsidérer les enjeux politiques, économiques et sociaux qui concernent actuellement l'éducation (Conseil de la famille, 1995). Le partenariat familles-école est posé ici à la fois comme une question de fond et comme une prémisse indis- pensable à toute évolution de l'institution scolaire.

Dans ce contexte, le partenariat avec les familles défavorisées représente un défi supplémentaire pour l'école québécoise. En effet, d'un côté on note l'absence manifeste des représentants de ce milieu dans les instances actuelles de participation (conseils de parents, d'orientation, comités d'école), alors que leur implication dans l'école est, par ailleurs, identifiée comme une modalité essentielle de l'amélioration des performances scolaires des enfants jugés les plus vulnérables (Bouchard, 1991).

\section{Deux stratégies partenariales de l'école}

Reposant sur la critique, déjà ancienne, de la fermeture de l'école aux cultures et vécus familiaux (Laperrière, 1983) et sur la logique contemporaine du partenariat, la question de la complémentarité ou de la concurrence entre les familles et l'école reste centrale dans les débats actuels sur l'éducation. Par exemple, le Conseil supérieur de l'éducation (1994) se donne pour objectif de «considérer la famille comme lieu d'accompagnement et de renforcement du processus scolaire » alors que le Conseil de la famille (1995) insiste sur «la responsabilité première de la famille dans l'éducation des enfants et sur le nécessaire accompagnement des familles par l'éducation scolaire ». La volonté partenariale de l'école s'inscrit ainsi dans ces discours contradictoires dans le même temps qu'elle est, comme toute orientation institutionnelle, régulée par les logiques sociales prépondérantes tant au Québec que, plus globalement, dans le monde occidental. Fondant notre perspective sur l'analyse d'une vingtaine de projets partenariaux d'écoles primaires québécoises avec les familles de leurs élèves ${ }^{1}$, nous identifions deux rationalités qui nous semblent cen- 
trales dans ces projets. La première repose sur la logique de la rationalisation scientifique et de la technocratisation des savoirs (Dandurand et Ollivier, 1991), la seconde sur la tendance à la diffusion et à l'élargissement des missions sociales de toutes les institutions, dont l'école (Lesemann et Chaume, 1992). Nous proposons, de ce fait, l'analyse et l'illustration de deux stratégies partenariales de l'école s'articulant plus spécifiquement sur l'une ou l'autre de ces logiques. La notion de stratégie sera, dans cette première partie, définie comme une orientation institutionnelle et organisationnelle qui conduit l'action tout en induisant des effets attendus et des effets pervers (Crozier, 1977). Dans les deux cas, le partenariat avec les familles est envisagé selon la rationalité à l'œuvre dans l'éducation scolaire donnée aux enfants et se construit sur des conceptions différentes de l'éducation, de l'école et de la famille.

\section{La stratégie d'apprentissage des compétences}

Dans cette orientation, l'école découpe son champ d'intervention, d'action et de partenariat par compétences. Cette notion est d'abord devenue centrale dans les programmes scolaires du primaire et du secondaire des années 1980 puis elle a gagné les CEGEP par la réforme de 1993-1994 (Roy, 1994). Il s'est alors agi d'identifier et de catégoriser les apprentissages des enfants en performances, habiletés et compétences avant tout observables mais aussi mesurables et évaluables. Bien que ce découpage pointu par compétences morcelées soit remis en question par de nombreux enseignants (Lemaire, 1996), il est actuellement la norme qui structure les programmes et la pédagogie. Mais bien plus, c'est cette même logique qui préside à certaines tentatives de partenariat entre l'école et les familles. Ainsi le
Conseil supérieur de l'éducation mentionne, en 1994, les très nombreuses et très spécifiques compétences à acquérir par les parents d'élèves: entre la nécessité d' "apprendre à structurer autrement le temps » et celle de «s'habiliter à transiger avec le personnel enseignant ", on trouve celle de «s'outiller pour encadrer les devoirs adéquatement » ou encore de «comprendre la culture scolaire» et de «réagir adéquatement aux évaluations». Être parents d'élèves, nous disent les écoles des compétences, c'est passer du statut «d'éducateur naturel», terme employé par les organismes familiaux, à celui d'éducateur parascolaire, maîtrisant des outils quasi professionnels et favorisant avant tout l'adaptation de l'enfant à la logique scolaire. Dans cette stratégie, le parent entre en apprentissage de ses nouvelles habiletés de parent d'élève au moment où l'enfant entre à l'école et souvent avant.

Les projets de formation des parents pour l'aide aux devoirs représentent une bonne illustration de cette orientation. Dans plusieurs programmes, c'est le personnel scolaire (professeurs, psycho-éducateurs et psychologues de l'école) qui donne la formation aux parents. Les compétences sont ainsi non seulement très découpées, elles sont aussi professionnalisées et rationalisées par la logique scolaire. De plus la pédagogie de ces groupes ressemble fort à celle de l'école: les parents doivent faire des devoirs chez eux, ils apprennent par essais-erreurs, ils font de la résolution de problèmes tout comme leurs enfants dans leurs classes. Notons que des formations de ce type sont actuellement en croissance, centrées sur les différentes compétences qu'il faut acquérir pour être de bons parents d'élèves. Les unes s'intéressent aux «habiletés à développer pour parti- ciper aux comités de parents », d'autres ont pour objectif de «développer les connaissances sur l'école » et sont souvent proposées aux familles avant l'entrée de l'enfant en maternelle. Les apprentissages qui y sont visés découpent alors de manière encore plus précise les comportements parentaux : "Comment équiper mon enfant», "Que faire le jour de la rentrée», "Comment communiquer avec son professeur » $\mathrm{y}$ sont des thèrnes fréquemment retrouvés.

Mais plus encore, certains groupes scolaires, issus de cette même volonté partenariale, effectuent un glissement autour du type de compétences en jeu dans les formations. Partant de celles qui identifient un bon parent d'élève, ces écoles vont cheminer vers celles qui qualifient aujourd'hui un bon parent. Ainsi, de nombreux établissements, financés en cela par le ministère de l'Éducation (pensons à l'ex-plan Pagé ou aux enveloppes régionales des commissions scolaires) ou par le ministère de la Santé et des Services sociaux (les écoles agissent alors souvent en partenariat avec des organismes locaux publics ou communautaires), mettent en cuvre des groupes dits de compétences parentales. Le plus souvent proposés avant l'entrée à la maternelle aux familles diagnostiquées à risque, soit du fait de leur quartier de résidence $^{2}$, soit par les dépistages effectués dans les centres locaux de services communautaires ${ }^{3}$, ces groupes visent à favoriser l'adaptation de l'enfant à l'école en outillant d'avantage les parents (Terisse, 1988). La réussite scolaire est ici perçue comme conditionnée par l'éducation familiale. Les parents doivent dès lors apprendre à devenir des éducateurs efficaces, adaptés aux étapes du développernent, au tempérament et au style d'apprentissage de leurs enfants pour leur ouvrir la voie de la perfor- 
Les nouveaux partenariats famille-école au Québec l'extériorité comme stratégie de survie des familles défavorisées ?

mance scolaire. Là encore, si les habiletés visées sont le plus souvent d'ordre communicationnel, ces groupes sont en majorité axés sur l'apprentissage des normes et savoirs scientifiques qui tournent autour du développement et des besoins de l'enfant. Citons les programmes «Parents efficaces» (Gordon, 1979) ou «Y a personne de parfait » (Brochu, 1992), les plus anciens au Québec et au Canada, ou encore le récent «PassePartout » (Conseil supérieur de l'éducation, 1994), qui font le tour des écoles maternelles et primaires de la province. Passant des compétences spécifiques au scolaire à celles qui structurent la vie familiale, l'école rejoint alors le champ déjà fort occupé des experts de l'éducation familiale (Dandurand et Dulac, 1992).

La stratégie de l'apprentissage des compétences s'inscrit ainsi, à travers ses glissements, dans la logique de rationalisation, de parcellarisation et de normalisation des savoirs scolaires qui s'étend, peut-être de manière impérialiste, à la vie familiale (Dandurand et Ollivier, 1991). Dès lors, nous sommes en mesure d'identifier les effets pervers ou inattendus de cette stratégie selon la logique qui l'imprègne. Le premier concerne la professionnalisation des parents dans leurs fonctions éducatives, tant en lien avec l'école qu'en dehors d'elle. Cette tendance a aussi pour corollaire de dévaloriser ou de nier les habiletés ou façons de faire des familles au quotidien, les savoirs vernaculaires, ceux-ci étant présentés comme peu scientifiques par rapport aux savoirs d'experts de l'école (Lave, 1991). Le second effet identifié concerne l'accroissement de l'écart entre les familles de divers milieux, favorisé par cette stratégie. Les recherches sur la participation des familles à l'école démontrent depuis plusieurs années que ce sont les «bons parents » qui participent aux structures partenariales mises en place par l'école. Ces familles sont le plus souvent issues des classes moyennes ou favorisées, elles ont un bon niveau scolaire et entretiennent des rapports réguliers avec les professeurs de leurs enfants (Hohl, 1981). Nous croyons que, contrairement à leurs objectifs, les groupes de compétences risquent de renforcer cette dichotomie. En effet, les évaluations actuelles (Conseil de la famille, 1995) démontrent le bon niveau de participation des familles (entre 50 et 80 pour cent selon les cas), leur satisfaction élevée à la fin du groupe, mais soulignent aussi qu'il y a toujours une fraction des familles qui n'y viennent pas: ce sont les familles les plus défavorisées, qu'on ne sait toujours pas comment atteindre et qui, dans cette course aux compétences, se retrouvent encore plus au bas de l'échelle, l'apprentissage devenant la norme. C'est face à cette réalité constante de l'inégalité des enfants et des parents devant l'école et ses savoirs que s'est construit l'autre type de stratégie partenariale de l'école.

\section{La stratégie de l'intervention sociale}

C'est ici dans une volonté de démocratisation de la culture scolaire que l'école ouvre ses portes (Lessard, 1994). Se situant dans une perspective humaniste, l'insti-

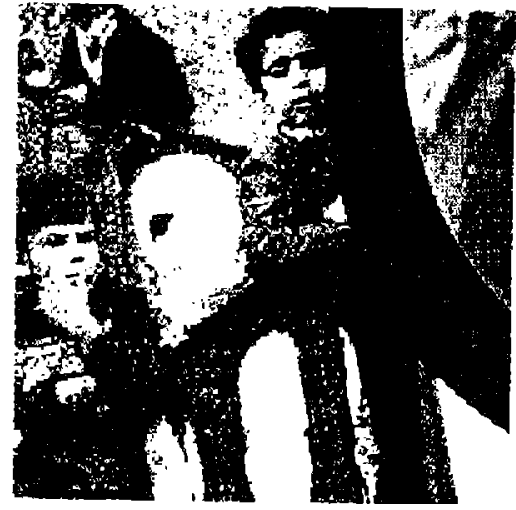

tution scolaire privilégie alors sa mission sociale: «former un individu pour la société contemporaine» (Dandurand et Dulac, 1992), ou encore faire de l'enfant un être social complet. Dans cette logique, on assiste à une transformation essentielle de l'école, auparavant analysée comme fermée à la famille et au social (Hohl, 1981) et se voulant désormais ouverte, humaine, empathique envers l'enfant et sa famille, selon les étapes et les problèmes de leur vie. Cette stratégie a ainsi pour objectif de répondre aux attentes des familles qui «souhaitent une école à leur service» (Conseil de la famille, 1995). Historiquement, cette ouverture a débuté, dans les années 1980, par les activités parascolaires (levées de fonds, sorties culturelles, bibliothèques et laboratoires informatiques) auxquelles les parents étaient conviés et qui devaient leur permettre d'apprivoiser l'école. Il s'agissait alors de faire du social avec les familles. Il semble bien que cette ouverture ait d'abord été une réussite parce que les parents participants étaient ceux qui partageaient le plus la culture scolaire, d'une part, mais aussi parce que cette collaboration, à la marge de l'éducation scolaire, n'était pas menaçante pour les professeurs (Laperrière, 1983). Dans un deuxième temps, l'école a élargi 
à la fois sa mission et les savoirs qu'elle veut transmettre, devenant une intervenante du social pour mieux dépister, adapter, aider les enfants qu'elle veut former. Dans cette stratégie, les savoirs valorisés par le monde scolaire s'éloignent de plus en plus des connaissances traditionnelles pour prendre la forme de normes et de valeurs importées à l'école par des acteurs et spécialistes du social (experts du développement de l'enfant, du couple, de la famille ou bien de leurs problématiques). Notons ici la multiplication, sur le temps et dans l'espace scolaires, de divers programmes de prévention ${ }^{4}$ auxquels participent les enfants et parfois leurs parents. Dans leur objectif de socialisation globale de l'enfant, certaines écoles tendent alors à devenir un espace social, ouvert et central pour la communauté. Afin de remplir cette large mission, et pour répondre à ce qu'elles identifient comme les besoins spécifiques des parents, ces institutions s'adjoignent alors des professionnels du social, experts de diverses problématiques (diététiciennes, infirmières, intervenants sociaux spécialistes des séparations parentales, de la communication familiale, de la violence familiale etc.) qui vont permettre l'entrée du social, et peut-être des familles dites défavorisées, à l'école. Nous pouvons ainsi nous référer à plusieurs projets partenariaux entre centres locaux de services communautaires et écoles qui, sur le modèle de l'école communautaire de Toronto (Bouchard, 1991), visent à organiser, dans l'école, des services pour les familles et les parents. Le projet d'école de développement de la santé globale de Sherbrooke est une illustration de ces partenariats multiples, conduits par l'école et visant à la prise en charge globale de la famille en son sein . Dans ces écoles ouvertes, les parents doivent pouvoir participer à certaines activités avec ou sans leurs enfants, et dans certains cas y régler leurs problèmes. L'espace scolaire est alors lieu d'adaptation et de socialisation pour les enfants comme pour les parents. De même, bon nombre de projets, proches de "la logique hygiéniste" de certaines écoles des banlieues françaises (Rogovas et Chauveau, 1993), visent à faire pénétrer les parents pauvres à l'école par le biais d'une aide alimentaire: ces établissements organisent, dans leurs locaux, des cuisines collectives qui ont pour objectifs d'apprivoiser les parents au monde scolaire et de les sortir de leur isolement tout en améliorant leurs compétences diététiques et parentales.

Former des enfants pour la société actuelle implique ainsi de reformer leurs parents aux normes et valeurs contemporaines. La stratégie de l'intervention sociale s'inscrit alors dans une logique où l'école est le seul filtre de la socialisation des enfants. Parfois vécue comme un débordement institutionnel sur la vie des familles, elle peut aussi être perçue comme une ingérence. Rappelons ici les réactions négatives des familles de milieu rural de Dandurand et Dulac (1992) face à l'écoute active des professeurs lorsqu'il y a un problème familial. Pensons aussi aux appropriations inattendues des possibilités ainsi ouvertes aux familles: des parents de milieu urbain défavorisé donnent à l'école un rôle supplétif de plus en plus large, d'autres sont portés à s'en remettre à elle pour tout régler, entrant dans une situation de dépendance (Roy, 1994), par exemple. Enfin, il nous paraît essentiel de noter que la stratégie d'intervention sociale véhicule l'idée d'une culture civique commune qui transcenderait les différences de classes et de familles. Reprenant comme norme d'éducation le modèle valorisé dans la classe moyenne québécoise, dont le dialogue, la communication et l'autonomie représentent les trois axes essentiels (Bouchard et Archambault, 1992), l'école sociale tend ainsi à marginaliser un peu plus les familles, sans doute minoritaires, qui ne le partagent pas.

Finalement, il ressort de ces deux stratégies de l'école deux effets sans doute contradictoires par rapport à leur intention partenariale: il s'agit du renforcement de l'écart entre les familles et de l'envahissement de la vie des familles par l'école. Qu'il s'agisse de la logique d'expertise présente dans la première stratégie ou de la diffusion de la mission scolaire centrale dans la seconde, il semble bien que ces effets soient particulièrement prégnants pour les familles les plus défavorisées sur les plans économique et socio-culturel. Ainsi, les familles de milieux défavorisés seraient à la fois les plus visées par les mesures partenariales de l'école et les plus soumises à leurs effets inattendus.

Ajoutons ici que toutes les recherches sur ces familles au Québec s'entendent pour parler de leur hétérogénéité, sur le plan de leur configuration (monoparentale, biparentale, reconstituée), de leurs sources de revenus (aide sociale, emplois précaires, aide intergénératïonnelle), de leur niveau de scolarité (qui va du diplôme universitaire à l'analphabétisme) ou encore de leur style d'éducation (Bouchard et Archambault, 1992 ; Fortin, 1987). Nous pouvons dès lors postuler qu'elles vont, de même, être fort diverses, non seulement dans leurs attentes mais aussi dans leurs appropriations et dans leurs réactions aux stratégies partenariales de l'école. Nous proposons pour notre part de comprendre et d'analyser ces appropriations diversifiées, constitutives de nouveaux rapports familles-école, au travers du concept de stratégie familiale de survie. 
LIEN SOCIAL ET POLITIQUES - RIAC, 35

Les nouveaux partenariats famille-école au Québec l'extériorité comme stratégie de survie des familles défavorisées?

92

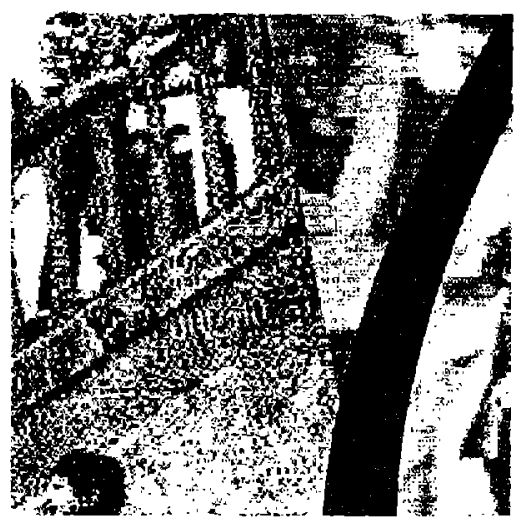

Les stratégies familiales de survie comme analyseur du rapport entre les familles et l'école

Il y a de nombreuses manières d'envisager les familles, leurs fonctionnements et leurs changements. $\mathrm{Si}$ l'approche systémique nous amène à les considérer comme des systèmes plus ou moins ouverts et en communication avec d'autres systèmes sociaux, la notion de réseaux sociaux (Brodeur, 1992) nous en donne une autre topographie. Elles sont alors reliées à certains réseaux socio-culturels et socio-économiques, et chacun de leurs membres participe aussi individuellement à des réseaux qui lui sont spécifiques. Dans une troisième approche, centrée sur les trajectoires de vie (Elder, 1977; Gaulejac, 1990), c'est l'histoire, la biographie sociale de la famille qui viennent donner sens aux comportements et pratiques des individus.

Dès lors, afin de mieux cerner la complexité des familles, nous proposons d'explorer l'interface psycho-sociale entre individus, familles et environnements par l'articulation de trois éléments: l'histoire ou expérience familiale, les réseaux sociaux de la famille et de ses membres et enfin la sousculture familiale. Cette interface remplit selon nous des fonctions de jonction, de lien et d'ajustement réciproque à deux niveaux : celui des individus et celui du nous familial (Hurtubise et Vatz Laaroussi, 1995). Cette notion d'interface nous renvoie au jeu qui s'élabore entre l'autonomie des agents et les contraintes des structures (Boudon, 1979), mais aussi à l'élaboration d'une zone d'incertitude ou de liberté (Crozier, 1977) dans laquelle sont générées, catalysées et opérationnalisées des stratégies individuelles et collectives. Ces stratégies sont à la fois produit, expression et élaboration de rapports dynamiques entre l'individu et la société et entre la famille et la société. Développée dans le champ des organisations (Crozier, 1977) puis de la psychologie culturelle (Camilleri, 1990) et de la sociologie clinique (Gaulejac et Taboada Leonetti, 1995), la notion de stratégie a été opérationnalisée sur les plans collectif puis individuel pour saisir les modalités d'adaptation, de changement et d'innovation mises en ouvre de manière consciente et inconsciente par les acteurs sociaux confrontés à des situations, à des événements et à des enjeux qu'ils ne maîtrisent pas entièrement. Ces stratégies font alors référence à un ensemble de représentations et de pratiques à la fois dynamiques et interactives. Elles sont le montage spécifique effectué par les individus ou les groupes entre leurs expériences, attitudes, représentations et pratiques d'ajustement aux environnements inventées au quotidien.

Notre première approche de la notion de stratégies familiales s'est effectuée en France auprès de chômeurs de longue durée (Vatz Laaroussi, 1988). Étudiant les modalités de survie quotidienne des familles soumises à une situation d'extrême précarité et à un processus social d'exclusion, nous avons pu saisir que les membres de ces familles mettaient en cuvre au jour le jour des moyens de survie tant matérielle que relationnelle et sociale. Travaillant avec les trajectoires familiales, nous avons découvert que ces façons de survivre pouvaient s'analyser comme des stratégies d'abord individuelles, puis matrimoniales et finalement familiales. Élargissant le concept de stratégie familiale d'articulation famille-travail développé par Pitrou (1987), nous les posons comme un analyseur des actions, représentations et pratiques des membres de la famille entre eux et au sein des regroupements sociaux dans lesquels ils se situent. Les stratégies familiales infléchissent ainsi pour chaque membre de la famille, adultes et enfants, l'occupation du temps et de l'espace (Hurtubise et Vatz Laaroussi, 1995), les modalités de communication privilégiées, les pratiques d'adaptation à l'inconnu, la lecture du social et les modalités de l'innovation. Elles sont à l'articulation des modalités de protection et de changement mises en ouvre par chacun au sein du groupe familial. Dans le cadre des fonctions de survie qu'elles remplissent, elles sont le moyen familial d'exister et, peut-être, d'être reconnu en tant que groupe social actif, original et participatif. Elles sont dans le même temps une modalité d'insertion sociale pour les individus membres de la famille, ici vus comme autonomes, acteurs du social et de leur trajectoire. Enfin, cette perspective des 
stratégies familiales nous permet d'articuler de manière dialectique le regard diachronique (la trajectoire avec ses ruptures et ses virages comme modalité d'ancrage dans l'histoire sociale) et le regard synchronique (le quotidien et la continuité comme modalités d'insertion et d'action dans le champ social) portés sur les familles. Nous croyons dès lors que, colorant et modelant les savoirs, savoir-faire et savoir-être des divers membres de la famille, ces stratégies visant à la survie de la famille et des individus qui la constituent nous permettent d'appréhender de manière originale le rapport des familles, parents et enfants, à l'institution-école, à la culture scolaire et aux savoirs et stratégies de l'école.

Une étude exploratoire menée en 1992-1993 auprès de familles dites défavorisées de Sherbrooke au Québec ${ }^{5}$ nous permet de postuler trois types de stratégies familiales de survie en contexte régional québécois (Vatz Laaroussi, 1994). Les familles sur lesquelles cette analyse a porté avaient toutes des enfants suivis en intervention précoce en milieu scolaire, c'est-à-dire qu'elles avaient déjà été dépistées et diagnostiquées comme à risque pour leurs enfants. Elles conjuguaient ainsi une pauvreté structurelle (la plupart des parents étaient sans emploi, souvent depuis plusieurs années, ou multipliaient, dans une trajectoire de précarité, des emplois successifs mal rémunérés; beaucoup percevaient le bien-être social), un faible capital scolaire (la plupart étaient de jeunes parents ayant décroché dans les premières années du secondaire), un réseau relationnel peu connu et reconnu des structures sociales et une forme d'exclusion sociale délimitée à la fois par leur quartier de vie (une des zones grises de la commission scolaire) et par leurs rapports à la société sou- vent réduits à l'étiquetage institutionnel. L'intervention précoce auprès des enfants de quatre ans ayant pour principal objectif de réparer un manque de stimulation familiale pour mieux préparer les enfants à l'école, ces familles sont avant tout connues pour leurs incapacités, leurs difficultés, leurs manques ou leurs carences. Pour notre part, nous avons voulu les regarder comme actrices dans leurs stratégies familiales de survie. Notre hypothèse est que, défavorisées, précarisées, dévalorisées et exclues, ces familles, puisqu'elles survivent comme entité matérielle et symbolique, entretiennent avec le social des rapports qui leur permettent à la fois de se protéger et de s'insérer, le plus souvent à la marge.

\section{L'expertise aux autres}

La première stratégie familiale identifiée, que nous nommerons «l'expertise aux autres», recouvre les pratiques et représentations des familles qui, soumises aux discours et pratiques d'expertise professionnelle, les intériorisent et se présentent comme incompétentes à se prendre en charge. Pour elles, la seule façon de survivre dans le monde d'experts que nous avons précédemment décrit, c'est de reconnaître les experts et de leur donner toute la responsabilité du devenir individuel et familial. L'expérience et les savoirs individuels et familiaux sont reniés, dévalorisés, étiquetés par la famille elle-même comme dysfonctionnements ou problèmes. Cet étiquetage devient, pour les membres de la famille, la porte d'entrée obligée à l'aide et à la reconnaissance sociale. Cette internalisation de l'incompétence se manifeste dans tous les domaines: vie quotidienne, relations à l'intérieur de et hors de la famille, prise en charge de la santé des adultes et des jeunes, domaine économique, vie profes- sionnelle et bien sûr éducation et scolarité des enfants.

Nous avions isolé, chez les familles de chômeurs de longue durée en France, une stratégie famiiale centrée sur la maladie comme seule modalité de survie économique et d'insertion sociale. De la même manière, certaines familles québécoises en viennent à se définir par leurs incompétences comme seul moyen d'obtenir une place dans le champ du social découpé par problématiques. Les membres de ces familles ne se situent alors que dans les rapports qu'ils entretiennent avec les divers experts de leurs problèmes de communication, d'estime de soi, de dévalorisation, d'apprentissage, d'inadaptation scolaire etc. Comme dans le processus de stigmatisation, ils prennent à leur charge le marquage social qui leur est imposé, mais plus encore, ils l'utilisent comme modalité d'insertion dans ce monde de l'expertise où leur place ne peut être que celle de l' «expertisé». C'est alors le médecin, le travailleur social, l'orienteur professionnel ou l'enseignant qui se voient chargés de découper la famille et ses membres en domaines d'incompétence à combler.

Finalement, la famille se représente à elle-même comme un ensemble morcelé de problématiques qu'elle utilise au fil de ses rapports sociaux pour, à la fois, se protéger et s'insérer. Cette stratégie de repli et peut-être d'impuissance face aux ingérences des experts devient aussi porteuse de tactiques conjoncturelles de résistance passive face à une société de plus en plus spécialisée. En ce qui concerne l'école, les parents la considèrent comme toute-puissante dans l'éducation de l'enfant, puisque experte tant sur le plan de l'instruction que sur le plan de la socialisation. Par contre, comme avec tous les savoirs d'expertise, ce sont des rapports de 
Les nouveaux partenariats famille-école au Québec : l'extériorité comme stratégie de survie des familles défavorisées?
94

distance qui s'instaurent avec l'école. Dès lors, et bien qu'inspirée par un même contexte normatif, cette stratégie familiale entre en opposition avec la stratégie d'apprentissage des compétences de l'école. Ici les parents, tout comme les enfants, n'envisagent pas de devenir compétents: bien que participant souvent aux groupes mis en œuvre par l'école, ils résistent à l'impérialisme de la logique de compétence en la laissant à l'extérieur de leur vie, de leurs espoirs, de leurs changements. Ces familles sont celles qui, peu revendicatrices dans ces groupes, s'en diront satisfaites puisqu'elles ont pu s'y faire reconnaître, mais celles aussi qui s'approprieront le moins les savoirs qui y sont véhiculés. Rester incompétentes est, pour elles, la seule manière de continuer à être reconnues des experts. Le rapport entretenu par les enfants avec l'école sera, lui aussi, distant, respectueux de ses savoirs mais peu enclin à les partager. De la même manière, ces familles sont peu perméables à la stratégie de l'intervention sociale qui veut articuler en son sein les différentes expertises. Là encore, l'extériorité familiale sera une stratégie de survie face à l'invasion diffuse de l'école. En particulier, les modèles éducatifs de communication et de compromis qui y sont valorisés entrent en opposition avec l'image carencée et problématique que les expertisés doivent donner d'eux-mêmes. Alors, si ces familles fréquentent l'école de l'intervention sociale, elles auront tendance à continuer à s'identifier par problématiques et par expertises, morcelant ainsi la mission sociale globale que s'attribue l'institution.

\section{La débrouillardise}

La seconde stratégie familiale identifiée est celle de la débrouillardise. En France, nous avions cerné les stratégies familiales des petits boulots et celles de la solidarité. Selon le contexte québécois, la «débrouille», analysée aussi par Grell et Wery (1993) auprès d'assistés sociaux, recouvre pour les adultes et les enfants de la famille petits boulots au noir, affiliation à des groupes divers d'entraide (cuisines collectives, paroisses, paniers de Noël, regroupements d'assistés sociaux, de familles monoparentales, de quartiers défavorisés) et solidarité familiale intergénérationnelle. Certaines des modalités de la débrouille seront à la frontière de la légalité (fausses déclarations pour augmenter le taux du bien-être social), d'autres beaucoup plus traditionnelles (il en est ainsi pour la circulation d'argent entre générations), mais toutes auront pour objet et résultat d'assurer à la fois la survie économique et la cohésion de la famille.

Contrairement à la stratégie précédente, la «débrouille » implique l'utilisation conjoncturelle des savoirs individuels et familiaux, la polyvalence sur le plan des activités et la diversification tant des talents que des relations. Elle implique aussi une forme de liberté dans les contraintes de la précarité, et souvent les familles, par justification ou par remise en cause de la hiérarchie sociale, mettent en œuvre un rapport ludique à leur réalité difficile. Par contre, il y a ici une non- reconnaissance des expertises extérieures vues comme non opérationnelles face à la situation matérielle de la famille. La famille «bricole» avec les normes sans s'y opposer mais en ne leur accordant pas la légitimité des savoirs transmis par l'expérience. Ces stratégies, à la marge sans doute, peuvent être comprises comme des modalités d'évitement de la réalité, de contournement ou de marginalisation (Gaulejac et Taboada Leonetti, 1995), mais elles peuvent aussi être vues comme des formes de participation sociale et peut-être d'innovation (Duvignaud, 1980). Ici, bien sûr, le savoir scolaire est considéré comme non pertinent et les stratégies de partenariat des écoles sont perçues comme des intrusions, des ingérences dans les trajectoires familiales. Ces familles ne sont pas prêtes à se laisser envahir, ni par les compétences portées par l'école, ni par le social scolarisé. Elles seront les éternelles absentes des groupes de parents, des comités d'école, des activités socio-culturelles. Ou bien encore elles en feront un usage inattendu et hors-norme: citons ce père de famille opposé à toutes les expertises scolaires mais qui, accompagnant des enfants lors d'une levée de fonds, leur faisait connaître, hors des murs de l'école, les plaisirs de la liberté, à la limite de la légalité, pour le plaisir de « rire avec eux».

Non reconnues dans leurs spécificités, ces familles ne reconnaissent à l'école ni un savoir particulier, ni une fonction particulière. Les stratégies de débrouillardise portées par les enfants seront souvent vues comme des marques d'inadaptation, de manque de stimulation ou encore de retard de développement. Pensons aux enfants dits en retard parce qu'ils n'ont jamais feuilleté de livres dans leur famille mais qui participent régulièrement à la gestion de l'économie familiale. Ces enfants, 
marginalisés, seront rapidement orientés vers les experts de l'inadaptation, qui ne feront que renforcer et légitimer le malentendu de base sur les savoirs des uns et des autres.

\section{L'entrée dans la compétition}

Enfin, les stratégies dites d'«entrée dans la compétition» recouvrent des pratiques familiales et individuelles visant à s'insérer socialement dans une démarche individualiste de compétition, en valorisant les savoirs extérieurs sans pour autant renier les habiletés de l'expérience et les savoirs acquis familialement. Il s'agit alors d'utiliser les habiletés valorisées socialement, de la manière la plus fonctionnelle et pour «arriver premier»... à l'école, dans les loisirs, dans le travail, dans I'implication communautaire. C'est la logique individualiste de la concurrence et du néo-libéralisme qui préside à cette stratégie, mais il est surprenant de voir qu'elle n'est pas seulement utilisée de manière tactique par des individus, mais bien comme une philosophie de l'entité familiale. Enfants et parents prennent alors part à la compétition dans tous les domaines qui leur sont ouverts, par l'apprentissage de savoirs institués mais aussi par leur participation mesurée à tous les groupes, rencontres, projets etc. Ainsi, tous les trucs, toutes les recettes, toutes les techniques sont bons pour arriver premier, et les membres de ces familles sont des adeptes des formations en tous genres, tout comme des groupes de soutien ou de croissance personnelle. Nous n'avions pas trouvé cette stratégie chez les chômeurs de longue durée français, sans doute trop et depuis trop longtemps désinsérés pour espérer encore entrer dans la compétition sociale. Par contre, cette stratégie familiale visant à la promotion de la famille et de ses membres par la compéti- tion est très présente chez les familles immigrantes que nous avons approchées dans d'autres recherches (Vatz Laaroussi, 1993) tant en France qu'au Québec. La notion d'effort individuel et familial est alors centrale comme modalité de participation sociale et comme mesure de la place occupée. Effort, compétition, activisme, apprentissages et multiplication des tâches sont ainsi les valeurs qui, à la fois, génèrent le rapport au social et gèrent la négociation du quotidien familial.

Parents et enfants abordent la vie, et donc l'école, comme un combat que l'on gagne en s'y montrant le plus fort, le plus engagé, le plus apprenant etc. Dans ce type de stratégie, l'école de l'intervention sociale et l'école d'apprentissage des compétences semblent avoir leurs entrées. En tous cas, ces familles vont être très présentes dans le processus partenarial, quel qu'il soit. Elles participeront aux groupes de compétences, seront toujours prêtes pour une levée de fonds ou un accompagnement socio-culturel, deviendront vite responsables des groupes de cuisine collective etc. Cependant, les attentes de ces familles vont reposer sur la pleine reconnaissance de leurs efforts et de leurs performances participatives pour une réelle promotion sociale, alors que l'école, si elle parle maintenant d'adaptation ou de fonctionnement global social, semble abandonner le discours de la promotion et vouloir se situer dans les à-côtés de l'idéologie de la compétition.

Dès lors, malgré un champ de rencontre, celui des activités communes, attentes des familles et philosophies de l'école semblent encore «dissoner». Ainsi, voulant, par leur participation, entrer dans la compétition du monde scolaire, les parents se trouvent finalement en dehors de la logique de l'institution scolaire qui, par expertises ou diffusion de ses savoirs, ne les reconnaît pas comme objets de concurrence. Les enfants de ces familles, eux aussi dans un rapport compétitif, seront souvent considérés par l'école comme porteurs d'un handicap social structurel (essentiellement la pauvreté, parfois la configuration familiale) et donc à discriminer positivement. Les discours de l'égalité des droits et du néo-libéralisme s'opposeront ici de manière inattendue à travers les difficultés de communication entre une école sociale et des enfants individualistes.

\section{Les familles défavorisées et l'école : des rapports d'extériorité ou d'altérité?}

Finalement, bien des questions restent posées sur ces stratégies familiales. En particulier, si nous les avons identifiées dans des familles de milieu défavorisé, sontelles complètement absentes pour les familles de classe moyenne? Sont-elles spécifiques à certains types de configuration familiale? En quoi le niveau de scolarité des parents ou leur trajectoire socioprofessionnelle influencent-ils la mise en cuvre de l'une ou de l'autre? Une famille pourrait-elle changer de stratégie de survie au cours de sa trajectoire et quels seraient les déclencheurs de ces modifications? Nous croyons que des recherches plus systématiques sont à mener pour mieux comprendre à la fois les stratégies des familles et leurs imbrications avec les logiques scolaires. Cependant, les trois stratégies présentes chez les familles de milieu défavorisé québécois tendent, nous semblet-il, à instaurer un rapport de distance et d'extériorité à l'école comme seul moyen de survie face à ses orientations et tactiques impérialistes. En effet, qu'il s'agisse de donner l'expertise aux autres, de compétitionner dans la marge de ces expertises ou encore 

'extériorité comme stratégie de survie des famille défavorisées?

de se débrouiller dans les à-côtés sociaux, il est avant tout question de continuer à exister comme famille, comme individu, comme citoyen, avec ou sans l'école. Face à l'envahissement de l'école par ses compétences ou par son intervention sociale, les familles dites de milieu défavorisé sont à la fois proactives dans la mise en ouvre de leurs positions et stratégies, et réactives et défensives dans des tactiques visant à chercher d'autres reconnaissances, d'autres espaces d'insertion et d'autres valeurs. Finalement, pour nombre de ces familles non reconnues dans leurs savoirs et dans leur rapport au savoir, rester extérieures à l'école est le seul moyen de ne pas être avalées par une institution qui élargit peu à peu son champ de compétences. Ce rapport d'extériorité est alors une stratégie de survie pour la famille, quelles que soient par ailleurs les modalités de son expression. Cependant, cette extériorité n'est pas foncièrement opposée au partenariat famillesécole, elle en représente plutôt, pour certaines familles, une modalité, une appropriation ou peut-être une étape. Et voici reposée sous un autre angle la question de ce partenariat! Quel intérêt ces familles marginalisées et non reconnues y ont-elles? En quoi leurs enfants y gagnent-ils? Nous croyons que la transformation de ce rapport d'extériorité en rapport d'altérité, permettant une volonté et une réalité partenariales réciproques, ne pourra s'effectuer que par la reconnaissance des façons de faire, des savoirs, des cultures, des identités et des stratégies familiales. Cette reconnaissance passe avant tout par la promotion des familles, dans leur diversité et dans leur originalité. $\mathrm{Ne}$ s'agit-il pas, finalement, de concevoir les familles comme un espace d'insertion et de citoyenneté, au même titre que l'école peut être un espace de développement et de promotion sociale?

Michèle Vatz Laaroussi Département de service social Université de Sherbrooke

\section{Notes}

1 Ces projets ont été implantés dans les écoles de différentes régions du Québec. Les uns sont expérimentés essentiellement dans des quartiers de Montréal, ville cosmopolite où se côtoient des populations issues de divers milieux socio-économiques québécois et les membres des communautés ethniques. D'autres se développent dans des régions urbaines, semi-urbaines ou rurales, souvent en situation socio-économique précaire. Dans le cadre d'une étude non subventionnée, nous avons analysé des projets mis en œuvre dans des écoles de Montréal et de régions semiurbaines comme l'Estrie et la Mauricie. Notre corpus a consisté principalement en textes écrits (présentations de ces projets, guides d'animation et rapports d'évaluation). En Estrie, nous avons pu l'enrichir de nos observations lors de rencontres multipartenariales de concertation, soit dans des écoles, soit dans des commissions scolaires.

2 Nous faisons ici référence aux zones grises déterminées pour chaque commission scolaire selon le taux de diplomation et l'échelle des revenus de la population.

3 Il s'agit de bilans effectués à trois ou quatre ans, qui visent à mesurer le développement global de l'enfant ainsi que les stimulations dont il fait ou non l'objet.

4 Nous pouvons citer les très nombreux programmes de prévention de la violence, des abus, des toxicomanies, mais aussi ceux qui font la promotion des bonnes stratégies de communication avec les parents ou encore des relations pacifiques.
5 Celte étude a été effectuée dans le cadre du Collectif d'études et de recherche en petite enfance de l'Université de Sherbrooke et plus spécifiquement au sein d'un projet de concertation avec le milieu scolaire, le Programme d'intervention précoce.

\section{Bibliographie}

BOUCHARD, C. 1991. Un Québec fou de ses enfants. Rapport. Gouvernement du Québec.

BOUCHARD, J.-M., et J. ARCHAMBAULT. 1992. «Famille et modèles éducatifs : modes d'exercice du pouvoir ", dans Comprendre la famille. Actes du ler symposium québécois de recherche sur la famille. Québec, PUQ.

BOUDON, R. 1979. Effets pervers et ordre social. Paris, PUF.

BROCHU, C. 1992. « Le programme "Y'a personne de parfait" au Québec. Une intervention préventive $"$, dans Comprendre la famille. Actes du ler symposium québécois de recherche sur la famille. Québec, PUQ.

BRODEUR, C. 1992. L'Intervention de réseaux, 20 ans d'expérience. Bourgneuf la forêt, L'ARPE.

CARISSE, C. 1974. La Famille : mythe et réalité québécoise. Québec, Conseil des affaires sociales et de la famille.

CAMILLERI, C., J. KASTERSZTEIN, M. LIPIANSKY, $H$. MALEWSKA-PEYRE, I. TABOADA-LEONETTI et A. VASQUEZ. 1990. Stratégies identitaires. Paris, PUF

CONSEIL DE LA FAMILLE (Québec). 1995. L'École et les familles: de son ouverture à leur implication. "Penser et agir famille», no 7.

CSE (Conseil supérieur de l'éducation). 1994. $\hat{E}$ tre parent d'élève du primaire : une tâche éducative irremplaçable. Avis au Ministre de l'Éducation. Gouvernement du Québec.

CROZIER, M. 1977. L'Acteur et le système. Paris, Seuil.

DANDURAND, R. B., et F.-R. OUELLETTE. 1995. «Famille, état et structuration d'un champ familial ", Sociologie et sociétés, XXVII, 2 (automne).

DANDURAND, R. B., et G. DULAC. 1992. «Les nouvelles familles et l'école ", dans Comprendre la famille. Actes du Ier symposium québécois de recherche sur la famille. Québec, PUQ.

DANDURAND, P., et É. OLLIVIER. 1991. "Centralité des savoirs et éducation: vers 
de nouvelles politiques ", Sociologie et sociétés, XXIII, 1 (printemps).

DE GAULEJAC, V., et I. TABOADA LÉONETTI. 1995. La Lutte des places. Paris, Reconnaissances Hommes et perspectives.

DE GAULEJAC, V. 1990. Femmes au singulier ou la parentalité solitaire. Paris, Klincksieck.

ELDER, G. 1977. «Family History and the Life Course », Journal of Family, 2, $4: 279-304$.

GRELL, P., et A. WERY. 1993. Héros obscurs de la précarité. Paris, L'Harmattan.

HOHL, J. 1981. Les Enfants n'aiment pas la pédagogie. Montréal, PPFM-UQAM.

HURTUBISE, R., et M. VATZ LAAROUSSI 1995. « Histoires de familles et espaces : lorsque des familles se racontent », Cahiers de sociologie de la famille, no 1 , Les Espaces de la famille.

JASMIN, C. 1992. Plus qu'une loi. Rapport, Québec.

LAPERRIÈRE, A. 1983. L'Intégration socioscolaire des enfants immigrants dans les écoles de milieux socio-économiquement faibles : une recherche exploratoire. Montréal, Conseil scolaire de l'île de Montréal.

LAVE, J. 1991. «Acquisition des savoirs et pratiques de groupe ", Sociologie et sociétés, XXIII, 1 (printemps).

LEMAIRE, P. 1996. “Du pareil au même », Nouvelles $C E Q$, janvier-février.

LESEMANN, F., et C. CHAUME. 1992. Familles-providence. La part de l'État. Montréal, Éditions St-Martin.

LESSARD, C. 1994. «La scolarisation, du déterminisme triomphant à l'utilitarisme stratégique », dans Fernand DUMONT, Simon LANGLOIS et Yves MARTIN, dir. Traité des problèmes sociaux. Québec, IQRC.

MSSS (Ministère de la Santế et des Services sociaux du Québec). 1992. La Politique de la santé et du bien-être.

PITROU, A. 1987. «L'interaction entre la sphère du travail et la sphère de la vie familiale », Sociologie et sociétés, IX, 2 : 103-113.

PROULX, J.-P. 1994 «L'analphabétisme », dans Fernand DUMONT, Simon LANGLOIS et Yves MARTIN, dir. Traité des problèmes sociaux. Québec, IQRC.

ROGOVAS-CHAUVEAU, E., et G. CHAUVEAU. 1993. "Banlieues: le rêve de l'excellence ", Autrement, 136, mars, série Mutations, Ainsi change l'école. Paris.

ROY, P.-E. 1994. Réforme ou maquillage? La réforme de l'éducation au Québec. Laval, Méridien.

ROUSSEL, L. 1989. La Famille incertaine Paris, Odile Jacob.
TERISSE, B., 1988. Environnement familial, intervention précoce et éducation familiale. Montréal, UQAM, GRASS.

VATZ LAAROUSSI, M. 1988. Les Chômeurs de longue durée : acteurs ou exclus? Tours, Université François Rabelais, mémoire de maîtrise en sociologie.

VATZ LAAROUSSI, M. 1993. « Stratégies familiales d'immigrants et intervention en interculturel », Service social, 42, 1.

VATZ LAAROUSSI, M. 1994. «Stratégies familiales : pour un travail social avec les familles ", dans Comprendre la famille. Actes du 2 e symposium québécois de recherche sur la famille. Québec, PUQ. 